https://helda.helsinki.fi

\title{
Pulmonary embolism location is associated with the co-existence of the deep venous thrombosis
}

\section{Sane, Markus A.}

2019-07

Sane , M A , Laukkanen , J A , Graner , M A , Piirilä , P L , Harjola , V-P \& Mustonen , P E 2019 , ' Pulmonary embolism location is associated with the co-existence of the deep venous thrombosis ' , Blood Coagulation and Fibrinolysis , vol. 30 , no. 5 , pp. 188-192 . https://doi.org/10.1097/MBC

http://hdl.handle.net/10138/323994

https://doi.org/10.1097/MBC.0000000000000813

cc_by_nc

acceptedVersion

Downloaded from Helda, University of Helsinki institutional repository.

This is an electronic reprint of the original article.

This reprint may differ from the original in pagination and typographic detail.

Please cite the original version. 


\section{Pulmonary embolism location is associated with the co-existence of the deep venous thrombosis Markus A. Sane ${ }^{a}$, Jari A. Laukkanen ${ }^{a, b, c}$, Marit A. Granér ${ }^{d}$, Päivi L. Piiriläe, Veli-Pekka Harjola ${ }^{\mathrm{f}, \mathrm{g}}$ and Pirjo E. Mustonen ${ }^{\mathrm{a}}$}

Multiple studies have shown that in approximately half of individuals with pulmonary embolism (PE), the deep venous thrombosis (DVT) is not evident at the moment of PE diagnosis. The underlying factors and the origin of PE in these patients are not completely understood: missed DVT, embolization of DVT in its entirety, or de-novo PE being possible explanations. The aim of this study was to evaluate the differences in PE patient with or without co-existing DVT. Sixty-three consecutive PE patients were included. Whole leg bilateral Doppler compression ultrasound was performed to all patients. The PE location and extension,

C-reactive protein, platelet count, hemostatic markers FV, FVIII, FXIIla, Fibrinogen, von Willebrand factor antigen,

thrombomodulin were assessed. Thorough clinical assessment including echocardiography and pulmonary function tests were performed upon arrival and seven months later. The mean age of the patients was 57 years (SD 17.3) and $33(52 \%)$ were women. Thirty-one patients $(49.2 \%)$ had coexisting DVT. The presence of DVT was associated with the proximal location of the PE (100\%), whereas none of the patients $(n=10)$ with exclusively peripheral PE had coexisting DVT. The PE extension, the measured hemostatic and inflammatory markers or the patient characteristics did not statistically differ between patients with isolated PE and PE

\section{Introduction}

It is assumed that most of the pulmonary embolisms (PE) originate from the thrombosis of the lower extremity veins [1,2]. Multiple modalities have been used in the search for co-existing thrombosis including compression ultrasound (CUS) [3,4], contrast media lower extremity computer tomography [5], and total-body magnetic resonance direct thrombus imaging technique [6]. Regardless of the method used the result has been the same, in nearly half of the patients with PE there is no sign of the thrombosis apart from pulmonary arteries.

There is indirect evidence indicating that PE is not always a sequel of deep venous thrombosis (DVT). For example patients with underlying lung pathology such as asthma and chronic obstructive pulmonary disease (COPD), present more often with PE than with DVT $[7,8]$. Additionally, there is evidence that isolated PE and PE associated with DVT have differences concerning their prognosis and risk factors [9-11]. An example of the former is the Leiden $\mathrm{V}$ paradox; heterozygote factor $\mathrm{V}$ Leiden mutation is shown to be stronger risk factor for DV'T than for PE $[12,13]$. Also, the thrombus lysis time with co-existing DVT. In roughly half of the PE patients no DVT could be detected. The location of the PE was associated with the presence of co-existing DVT. There were no differences in the PE extension, hemostatic markers or in the patient characteristic between patients with isolated PE or PE with coexisting DVT. Blood Coagul Fibrinolysis 30:000-000 Copyright (C) 2019 Wolters Kluwer Health, Inc. All rights reserved.

Blood Coagulation and Fibrinolysis 2019, 30:00-00

Keywords: coagulation, deep venous thrombosis, hemostatic markers, isolated pulmonary embolism, pulmonary embolism

${ }^{a}$ Department of Internal Medicine, Jyväskylä Central Hospital, University of Eastern Finland, ${ }^{\mathrm{b}}$ Department of Clinical Medicine, University of Eastern Finland ${ }^{\mathrm{c}}$ Faculty of Sport and Health Sciences, University of Jyväskylä, Jyväskylä, ${ }^{\mathrm{d}}$ Heart and Lung Center, Cardiology, University of Helsinki and Helsinki University Hospital, ${ }^{e}$ Department of Clinical Physiology, HUS Medical Imaging Center, Helsinki University Central Hospital and University of Helsinki, ${ }^{\mathrm{f} D e p a r t m e n t}$ of Emergency Medicine, University of Helsinki and ${ }^{9}$ Department of Emergency Medicine and Services, Helsinki University Hospital, Helsinki, Finland

Correspondence to Markus A. Sane, MD, Töölönkatu 19a3 00260 Helsinki, Finland.

E-mail: markus.sane@fimnet.fi

Received 17 December 2018 Revised 26 March 2019 Accepted 10 April 2019

and the fibrin clot structure, as well as the profile of plasma hemostatic markers have been shown to differ between patients with isolated DV'T or isolated PE $[14,15]$ and patients with isolated PE and PE with DV'T [16]. Where the connection of pulmonary pathology and isolated PE seems plausible, less is known about the pathophysiology and background of isolated PE in patients without these underlying conditions.

The aim of this study was to assess the prevalence of isolated PE in consequent PE patients, from which underlying chronic pulmonary diseases were excluded both by medical history and comprehensive pulmonary function tests, and compare the features of PE, such as its location and extension, clinical findings, risk factors, and plasma levels of hemostatic markers FV, FVIII, FXIIIa, von Willebrand factor antigen (vWF:Ag), fibrinogen and C-reactive protein, in PE patients with and without co-existing DV'T.

\section{Methods}

This prospective study consisted of 63 consecutive patients with acute PE diagnosed in the Emergency 
Department of Helsinki University Hospital. The patients were enrolled between February 2003 and August 2004. The following exclusion criteria were applied: previous PE, preceding anticoagulation therapy, clinically high-risk PE requiring immediate thrombolysis, chronic pulmonary disease requiring regular medication, for example, COPD, unstable angina pectoris, heart failure, co-existing trauma, and terminal cancer.

The study design was approved by the Ethics Committee of the Helsinki University Central Hospital and written informed consent was obtained from all participants.

From this same patient cohort we have earlier published the radiologically calculated PE mass and its association to right ventricular function [17], and the comparison of hemostatic markers at acute phase and 7 months later, as well as the effect of VTE volume on these markers [18,19]. The diagnostics of $\mathrm{PE}$ and radiological calculation of $\mathrm{PE}$ mass have been described earlier in detail [17-19].

In brief, the diagnosis of the PE was confirmed with computer tomography pulmonary angiography (CTPA) and all scans were centrally assessed to evaluate the location and extension of PE by two expert radiologists blinded to other results. PE was considered to be proximal if it was located in the main, mediastinal or in the lobar pulmonary arteries, whereas the PE was classified as peripheral if the embolus was only in the segmental or subsegmental arteries. The most proximal location of the PE defined its category (proximal or peripheral).

The PE extension was evaluated with the method of Mastora, which is a semiquantitative method in evaluating the obstruction of the pulmonary arteries [20]. The Mastora score describes the combined PE mass by calculating the obstruction of the proximal (five mediastinal and six lobar arteries) and peripheral pulmonary arteries (twenty segmental arteries) using a 5-point scale. Each artery is evaluated separately and the sum of the total obstruction is reported in percentages both proximally and peripherally, maximum obstruction percentage being $155 \%$. If the $\mathrm{PE}$ is only in subsegmental artery the count is 0 .

Bilateral lower extremity Duplex US evaluation was performed to all patients with standard $10 \mathrm{MHz}$ linear array probe. The protocol for the diagnosis of DVT included compression of the common femoral, superficial femoral, popliteal, posterior tibial and peroneal veins. Compression was made every $3 \mathrm{~cm}$. Additionally Doppler US was performed at the junction of common femoral and great sapheus vein to assess the existence of more proximal venous thrombosis.

Majority of the patients $(47 / 63,74.6 \%)$ underwent thorough pulmonary testing including spirometry, pulmonary single breath diffusing capacity for carbon monoxide and measurement of fractional exhaled nitric oxide (FENO). The details of pulmonary function tests have been published earlier [21-22].
Twenty-eight patients had received single dose of lowmolecular-weight-heparin before the CTPA due to the high clinical suspicion of the PE. No other treatment was given before PE diagnosis or CUS. The choice of investigated hemostatic parameters, the sample collection, and the analysis of the hemostatic markers in national reference laboratories were performed as described earlier $[17,19]$. All individuals were also tested for thrombophilia.

All statistical analyses were performed with SPSS 21.0 for Windows (SPSS Inc., Chicago, Illinois, USA). Data are presented as means (with SD for normally distributed continuous variables) and proportions (\%). The normality of continuous variables was checked by the KolmogorovSmirnov test. Logarithmic transformation of variables was performed, if necessary.

Independent samples $t$ test was used in the analysis of the differences between hemostatic markers in patients with or without DVT. Cross-tabulation was used to evaluate the significance of the DVT effect on the location of the embolism and the effect of thrombophilia in patients with or without DVT. Fisher's exact test was used to measure the statistical significance due to the small sample size. A $P$ value $<0.05$ was considered statistically significant.

\section{Results}

The mean age of the patients was 57 years (SD 17.3) and $33(52 \%)$ were women. Thirty-two (50\%) had isolated PE. The DVTs were proximal in the majority of cases (25/31, $81 \%)$. There were no significant differences between PE patients with and without co-existing DVT regarding to the assessed patient characteristics and the common risk factors for venous thrombosis (Table 1). The demographic and clinical characteristics of the patients are presented in Table 1.

The association of the presence of DVT and PE location is presented in Table 2. All the patients with DVT $(n=31)$ had PE mass on proximal location (pulmonary truncus, main pulmonary or lobar pulmonary arteries) whereas none of the patients with exclusively peripheral PE $(n=10$, seven segmental and three subsegmental embolies) had co-existing DVT. The association of the DVT and PE location is presented in Table 2.

There was no difference in semiquantitatively evaluated total volume of PE (PE mass) between the groups (Fig. 1). Total Mastora score was 45.2 vs. 57.8 , peripheral Mastora score 30.3 vs. 39.0 and proximal score 14.9 vs. $18.8(P=0.13, P=0.17$ and $P=0.11$, respectively $)$. There were neither any statistically significant differences in the measured hemostatic or inflammatory markers between the two patients groups in the acute phase (Table 3 ) or when they were analyzed again seven months later (data not shown).

The volume (mass) of PE in patients with and without coexisting DVT expressed by Mastora score is presented in 


\begin{tabular}{|c|c|c|c|}
\hline & PE with co-existing DVT $(n=31)$ & PE without co-existing DVT $(n=32)$ & $P$ \\
\hline Age (years) [SD] & $57.3[17.1]$ & $55.7[17.6]$ & 0.7 \\
\hline Gender, male/female & $18 / 13$ & $19 / 13$ & 0.1 \\
\hline $\mathrm{BMI}\left(\mathrm{kg} / \mathrm{m}^{2}\right)[S D]$ & $29.7[5.4]$ & $29.3[5.6]$ & 0.7 \\
\hline Previous DVT, $n(\%)$ & $4(12.9)$ & $3(9.4)$ & 0.7 \\
\hline Hormone replacement therapy or oral contraception, $n(\%)$ & $6(19.4)$ & $9(28.1)$ & 0.5 \\
\hline Preceding immobilization, yes/no & $13 / 18$ & $19 / 13$ & 0.2 \\
\hline Current smoking (\%) & $5(16)$ & $5(16)$ & 0.9 \\
\hline Chronic pulmonary disease $^{a}$ & 0 & 0 & \\
\hline Fractional exhaled nitric oxide (ppb) (FENO) & $24.2[36.3](n=24)$ & $16.5[9.9](n=23)$ & 0.3 \\
\hline Echocardiographic sign of right ventricular dysfunction on admission, $\mathrm{n}(\%)$ & $18(58)$ & $14(44)$ & 0.3 \\
\hline Symptoms duration ( $\leq 1$ week or $>1$ week), yes/no & $13 / 18$ & $9 / 23$ & 0.1 \\
\hline Thrombofilia (all thrombophilia cases were heterozygous for factor $\mathrm{V}$ variant), $n$ & 4 & 1 & 0.3 \\
\hline
\end{tabular}

DVT, deep venous thrombosis; FENO, fractional exhaled nitric oxide; PE, pulmonary embolism. ${ }^{\text {a }}$ The clinical data of pulmonary disease requiring regular medication was exclusion criteria. Additionally majority of the patients (75\%) underwent thorough pulmonary testing including spirometry and diffusion capacity measurements. There were neither signs of parenchymal pulmonary disease in CT scan.

Fig. 1. Hemostatic and inflammatory markers in patients with or without co-existing DVT on acute phase are presented on Table 3.

\section{Discussion}

In our population of 63 consecutive PE patients without underlying lung pathology, isolated PE was as common as PE with DVT. PEs with DVT were more proximal than isolated PEs. None of the ten patients with exclusively peripheral PE (PE only in segmental or subsegmental arteries) had DVT. There were no statistically significant differences in patient characteristics or analyzed hemostatic and inflammatory markers between groups with DVT-associated and isolated PE. Interestingly, the total mass of PE was similar in both groups.

The share of isolated PEs $(32 / 63,50 \%)$ in our study is in line with several previous reports showing the absence of lower extremity DVT in $40-56 \%$ of newly diagnosed PE patients [3-6]. Isolated $\mathrm{PE}$ has probably multiple different etiologies or explanations. We hypothesized that if at least part of isolated PEs would be a different entity, characterized by in situ thrombus formation and triggered by local pulmonary vasculature reactive state, it would be more often peripheral than PE with concomitant DVT. Previously it has been shown, that patients with underlying thoracic pathology, such as asthma or COPD, present more often with PE than with DVT $[7,8]$. For example, in a study of Dutch asthma outpatient clinic, the incidence of PE in severe asthma was more than fivefold, and in mild-moderate asthma two-fold higher than in the general population, whereas asthma was not associated with the DVT [8]. The over presentation of PE in

\begin{tabular}{lcccc} 
Table 2 & The association of the DVT and PE location & \\
$\begin{array}{lccc}\text { Co-existing } \\
\text { DVT }\end{array}$ & $\begin{array}{c}\text { Proximal and } \\
\text { peripheral PE }\end{array}$ & $\begin{array}{c}\text { Exclusively } \\
\text { peripheral PE }\end{array}$ & $\begin{array}{c}\text { Total } \\
(\%)\end{array}$ & $P=0.001$ \\
\hline Yes (\%) & $31(49.2)$ & $0(0)$ & $31(49.2)$ & \\
No (\%) & $22(34.9)$ & $10(15.9)$ & $32(50.2)$ & \\
Total $(\%)$ & $53(84.1)$ & $10(15.9)$ & $63(100)$ & \\
\hline
\end{tabular}

DVT, deep venous thrombosis; PE, pulmonary embolism. asthma and COPD has been hypothesized to be linked to local vascular inflammation [23]. In our population, previously diagnosed chronic pulmonary disease was an exclusion criteria and pulmonary function tests showed no signs of undiagnosed chronic lung pathology. The pulmonary airways were evaluated even by measuring FENO which is a good surrogate marker for eosinofilic inflammation in the airways [24]. The FENO values were similar in two patient groups and also the average FENO levels were within the normal range of healthy adults [24].

In our study population, PE with co-existing DVT was more centrally located. All the patients with DVT $(n=31)$ had proximal PE. None of the patients with exclusively peripheral PE $(n=10)$ had co-existing DVT. Similar results have been reported previously in few PE patient cohorts. In two relatively small studies by Velmahos and Van Gent $(n=46$ and $n=31)$ it was shown that among trauma patients with $\mathrm{PE}$ and co-existing DVT, $60-70 \%$ of the PEs were proximal whereas only $7-29 \%$ of the exclusively peripheral PE patients had coexisting DVT $[5,25]$.

Local inflammatory state, endothelial dysfunction and prothrombotic tendency might predispose to in situ formed isolated PE [26]. We found no significant differences in the analyzed hemostatic or inflammatory markers between the patients with isolated PE or PE with coexisting DVT. It is unlikely, however, that these markers measured from systemic venous circulation during acute $\mathrm{PE}$ with concomitant acute large-scale systemic upregulation of thrombotic and inflammatory mechanisms, could adduce signs of local inflammation and procoagulant potential. Either, no statistical differences was found in these markers in stable phase 7 months later. Contradictory to this, a previous study showed, that in stable phase after the anticoagulation was withdrawn, fibrinogen levels, endogenous thrombin potential was significantly higher, and smoking prevalence lower in patients with isolated PE when compared to PE with DVT [16]. The size of the study population in the Zabczyk et al. [16] 


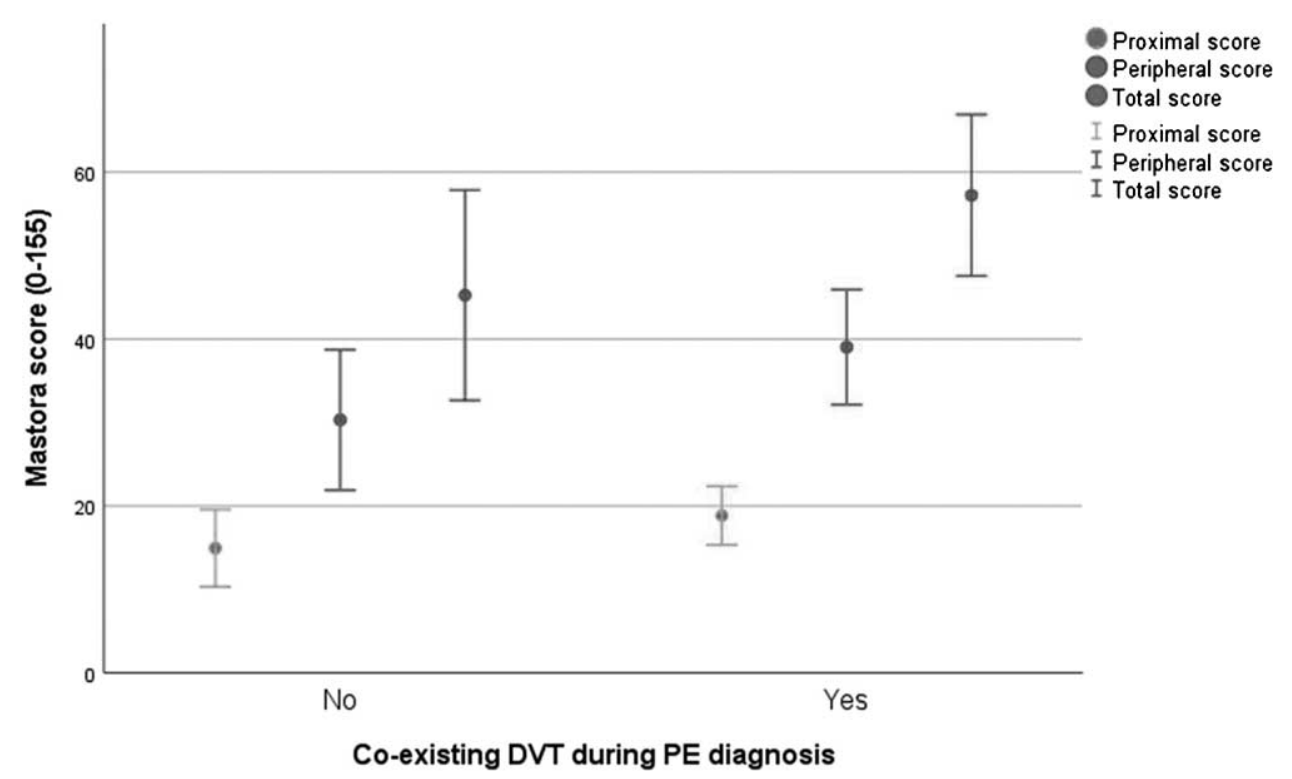

The volume (mass) of PE in patients with and without co-existing DVT expressed by Mastora score.

study was nearly threefold compared to our study and it might have more power to detect the statistically significant findings.

In addition to in-situ thrombosis, also other explanations for isolated PE exist. In patients with isolated PE, distal lower extremity DVTs might have been missed due to the limitations of CUS examination. The sensitivity of the whole leg Duplex US to detect proximal and distal DVT is 94 and 64\%, respectively [27]. Theoretically, embolism originating from small distal veins could as well end up into smaller peripheral pulmonary arteries. However, the distal DVTs are considered not to embolize as often as proximal ones [28] and although CUS examination misses $40 \%$ of them we consider unlikely that missed distal DVTs could explain that none of the patients with exclusively peripheral PE had co-existing DVT. Other undetected DVT locations are possible, too. The ability of Duplex US to detect pelvic vein thrombosis is

Table 3 Haemostatic and inflammatory markers in patients with or without co-existing DVT on acute phase

\begin{tabular}{lccc}
\hline $\begin{array}{l}\text { Hemostatic or } \\
\text { inflammatory marker }\end{array}$ & $\begin{array}{c}\text { PE with } \\
\text { co-existing DVT }\end{array}$ & $\begin{array}{c}\text { PE without } \\
\text { co-existing DVT }\end{array}$ & $P$ \\
\hline FV \% & $106[22.4]$ & $115[21.6]$ & 0.1 \\
FVIII \% & $165[56.7]$ & $170[55.9]$ & 0.7 \\
FXIIla \% & $82.4[18.8]$ & $92.5[29.1]$ & 0.1 \\
vWF:Ag (IU/ml) & $2.73[1.44]$ & $2.58[0.97]$ & 0.6 \\
C-reactive protein (mg/l) & $33.3[39.1]$ & $39.3[29.4]$ & 0.5 \\
Thrombomodulin $(\mathrm{ng} / \mathrm{ml})$ & $36.7[12.6]$ & $36.4[12.9]$ & 0.9 \\
Fibrinogen $(\mathrm{g} / \mathrm{l})$ & $4.4[1.5]$ & $4.1[1.3]$ & 0.4 \\
\hline
\end{tabular}

Data are presented as mean [SD]. DVT, deep venous thrombosis; PE, pulmonary embolism. uncertain. To enhance the sensitivity to detect iliac vein thrombosis, we used the Doppler waveform analysis [29]. We did not investigate the upper extremities. However, the prevalence of primary upper extremity DVT is rare, around two cases per 100000 person per year [30], and the most common causes of upper extremity DV'T are among exclusion criteria of the study. Rare cardiac origin for isolated PE, [31] is also most unlikely since all patients underwent CTPA and also echocardiography as described earlier [17] without signs of right ventricle thrombus. Consequently, we consider that missed DVTs are an unlikely explanation for the $50 \%$ share of the isolated PEs.

One possible explanation of isolated PE, although unknown in its prevalence, might be the embolization of DV'T in its entirely. Previous publications suggest that the free floating tail embolizes rather than the whole thrombus [32-34]. Moreover, it is unlikely that cases of entirely dislodged DV'Ts would end up more often in peripheral than in proximal pulmonary arteries.

Despite the limitations of US examination, the main limitation of our study is the relatively small patient cohort. However, the patient population was well characterized, profoundly investigated, and prospectively followed for 7 months. This allowed us to evaluate, that is, both the hemostatic parameters and pulmonary function tests also in the stable phase. It should be noted that the laboratory testing of serial hemostatic markers is costly and burdensome explaining the limited number of patients. 
In conclusion, we found that half of the PEs were isolated, even in the absence of any inflammatory lung disease. Isolated PE affected less often large pulmonary arteries, although its semiquantitatively assessed volume was not different form the PE associated with DVT. All of the exclusively peripheral PEs were isolated PEs. Peripheral in-situ pulmonary artery thrombosis is histopathologically evident in lung diseases like pulmonary hypertension [35] and acute respiratory distress syndrome [36], but to which extent it occurs in patients without underlying lung conditions, remains unclear. Further studies are needed to clarify the etiology of isolated $\mathrm{PE}$ in a population without lung pathology.

\section{Acknowledgements Conflicts of interest}

There are no conflicts of interest.

\section{References}

1 Kumar V, Abbas A, Aster J. Robbins and Cotran pathologic basis of disease. 9th ed. Philadelphia, PA: Elsevier Saunders.

2 Kakkar VV, Howe CT, Flanc C, Clarke MB. Natural history of post operative deep vein thrombosis. Lancet 1969; 2:230-232; 2.

3 Yamaki T, Nozaki M, Sakurai H, Takeuchi M, Soejima K, Kono T. Presence of lower limb deep vein thrombosis and prognosis in patients with symptomatic pulmonary embolism: preliminary report. Eur J Vasc Endovasc Surg 2009; 37:225-231.

4 Girard P, Sanchez O, Leroyer C, Musset D, Meyer G, Stern JB, et al. Deep venous thrombosis in patients with acute pulmonary embolism: prevalence, risk factors, and clinical significance. Chest 2005; 128:1593-1600.

5 Velmahos GC, Spaniolas K, Tabbara M, Abujudeh HH, de Moya M, Gervasini A, Alam HB. Pulmonary embolism and deep venous thrombosis in trauma: are they related? Arch Surg 2009; 144:928-932.

6 van Langevelde K, Šrámek A, Vincken PWJ, van Rooden JK, Rosendaal FR, Cannegieter SC. The origin of pulmonary emboli with a total-body magnetic resonance direct thrombus imaging technique. Haematologica 2013; 98:309-315.

7 Schneider C, Bothner U, Jick SS, Meier CR. Chronic obstructive pulmonary disease and the risk of cardiovascular diseases. Eur J Epidemiol 2010; 25:253-260.

8 Majoor CJ, Kamphuisen PW, Zwinderman AH, Ten Brinke A, Amelink M, Rijssenbeek-Nouwens L, Sterk PJ, et al. Risk of deep vein thrombosis and pulmonary embolism in asthma. Eur Respir J 2013; 42:655-661.

9 Kovacs MJ, Kahn SR, Wells PS, Anderson DA, Chagnon I, LE Gal G, et al. Patients with a first symptomatic unprovoked deep vein thrombosis are at higher risk of recurrent venous thromboembolism than patients with a first unprovoked pulmonary embolism. J Thromb Haemost 2010; 8:19261932.

10 Douketis JD, Gu CS, Schulman S, Ghirarduzzi A, Pengo V, Prandoni P. The risk for fatal pulmonary embolism after discontinuing anticoagulant therapy for venous thromboembolism. Ann Intern Med 2007; 147:766-774.

11 Gordon NT, Schreiber MA. Pulmonary emboli and deep vein thromboses: are they always part of the same disease spectrum? Mil Med 2016; 181 (5 Suppl):104-110.

12 Hirmerova J, Seidlerova J, Subrt I. The association of factor V Leiden with various clinical patterns of venous thromboembolism-the factor $\mathrm{V}$ Leiden paradox. QJM 2014; 107:715-720.

13 Zöller B, Melander O, Svensson PJ, Engström G. Factor V Leiden paradox in a middle-aged Swedish population: a prospective study. Vasc Med 2018; 23:52-59; 1358863X17745591.

14 Martinez MR, Cuker A, Mills AM, Crichlow A, Lightfoot RT, Chernysh IN, et al. Enhanced lysis and accelerated establishment of viscoelastic properties of fibrin clots are associated with pulmonary embolism. Am J Physiol Lung Cell Mol Physiol 2014; 306:L397-404.
15 Undas A, Zawilska K, Ciesla-Dul M, Lehmann-Kopydłowska A, Skubiszak A, Ciepłuch K, Tracz W. Altered fibrin clot structure/function in patients with idiopathic venous thromboembolism and in their relatives. Blood 2009; 114:4272-4278.

16 Zabczyk M, Plens K, Wojtowicz W, Undas A. Prothrombotic fibrin clot phenotype is associated with recurrent pulmonary embolism after discontinuation of anticoagulant therapy. Arterioscler Thromb Vasc Biol 2017; 37:365-373.

17 Granér M, Harjola VP, Selander T, Laiho MK, Piilonen A, Raade M, Mustonen P. N-Terminal pro-brain natriuretic peptide, high-sensitivity troponin and pulmonary artery clot score as predictors of right ventricular dysfunction in echocardiography. Heart Lung Circ 2016; 25:592-599.

18 Sane M, Granér M, Laukkanen JA, Harjola V-P, Mustonen P. Plasma levels of haemostatic factors in patients with pulmonary embolism on admission and seven months later. Int J Lab Hematol 2018; 40:66-71.

19 Sane M, Laukkanen JA, Granér M, Piilonen A, Raade M, Harjola VP, Mustonen $P$. Combined volume of pulmonary embolism and deep venous thrombosis-Association with FV, platelet count, and D-dimer. Int J Lab Hematol 2018; Epub ahead of print.

20 Mastora I, Remy-Jardin M, Masson P, Galland E, Delannoy V, Bauchart JJ, Remy J. Severity of acute pulmonary embolism: evaluation of a new spiral CT angiographic score in correlation with echocardiographic data. Eur Radiol 2003; 13:29-35.

21 Piirilä P, Laiho M, Mustonen P, Graner M, Piilonen A, Raade M, et al. Reduction in membrane component of diffusing capacity is associated with the extent of acute pulmonary embolism. Clin Physiol Funct Imaging 2011; 31:196-202.

22 Rouhos A, Kainu A, Piirilä P, Sarna S, Lindqvist A, Karjalainen J, Sovijärvi A. Repeatability of exhaled nitric oxide measurements in patients with COPD. Clin Physiol Funct Imaging 2011; 31:26-31.

23 Yan C, Wang X, Su H, Ying K. Recent progress in research on the pathogenesis of pulmonary thromboembolism: An old story with new perspectives. Biomed Res Int 2017; 2017:6516791.

24 Taylor DR, Pijnenburg MW, Smith AD, De Jongste JC. Exhaled nitric oxide measurements: clinical application and interpretation. Thorax 2006; 61:817-827.

25 Van Gent JM, Zander AL, Olson EJ, Shackford SR, Dunne CE, Sise CB, et al. Pulmonary embolism without deep venous thrombosis: de novo or missed deep venous thrombosis? J Trauma Acute Care Surg 2014; 76:1270-1274.

26 van der Poll T, Boer JD, Levi M. The effect of inflammation on coagulation and vice versa. Curr Opin Infect Dis 2011; 24:273-278.

27 Goodacre S, Sampson F, Stevenson M, Wailoo A, Sutton A, Thomas S, et al. Measurement of the clinical and cost-effectiveness of noninvasive diagnostic testing strategies for deep vein thrombosis. Health Technology Assessment 2006; 10:1-168.

28 Monreal M, Ruiz J, Olazabal A, Arias A, Roca J. Deep venous thrombosis and the risk of pulmonary embolism. A systematic study. Chest 1992; 102:677-681.

29 Kayılıoğlu SI, Köksoy C, Alaçayır İ. Diagnostic value of the femoral vein flow pattern for the detection of an iliocaval venous obstruction. J Vasc Surg Venous Lymphat Disord 2016; 4:2-8.

30 Lindblad B, Tengborn L, Bergqvist D. Deep vein thrombosis of the axillarysubclavian veins: epidemiologic data, effects of different types of treatment and late sequelae. Eur J Vasc Surg 1988; 2:161-165.

31 Prandoni P, Pesavento R, Sorensen HT, Gennaro N, Dalla Valle F, Minotto I, et al. Prevalence of heart diseases in patients with pulmonary embolism with and without peripheral venous thrombosis: findings from a crosssectional survey. Eur J Intern Med 2009; 20:470-473.

32 Coon WW, Coller FA. Clinicopathologic correlation in thromboembolism. Surg Gynecol Obstet 1959; 109:259-269.

33 Lindblad B, Sternby NH, Bergqvist D. Incidence of venous thromboembolism verified by necropsy over 30 years. BMJ $1991 ; \mathbf{3 0 2}: 709-711$.

34 Neyman M, Gewirtz J, Poncz M. Analysis of the spatial and temporal characteristics of platelet-delivered factor VIII-based clots. Blood 2008; 112:1101-1108.

35 Bazan IS, Fares WH. Hypercoagulability in pulmonary hypertension. Clin Chest Med 2018; 39:595-603.

36 Tomashefski JF, Davies P, Boggis C, Greene R, Zapol WM, Reid LM. The pulmonary vascular lesions of the adult respiratory distress syndrome. $A m \mathrm{~J}$ Pathol 1983; 112:112-126. 